\title{
Relationship of Airway Epithelial Ion Transport to Chronic Bronchitis
}

\author{
Richard C. Boucher \\ Cystic Fibrosis/Pulmonary Research and Treatment Center, University of North Carolina at Chapel Hill, Chapel Hill, North Carolina
}

\begin{abstract}
Failure to clear mucus normally is a critical event in the pathogenesis of chronic bronchitis (CB). A unifying concept for the failure to clear mucus from airway surfaces has emerged that focuses on an abnormally high mucin-to-airway surface liquid volume ratio. At a certain level, a high mucin-to-volume ratio is associated with loss of the periciliary liquid layer and adhesion of the mucus layer to the cell surface. Adherent mucus becomes a stimulus for irritation and cough and is the nidus for bacterial infection. Therapeutic approaches for clearing mucus from CB airways should focus on restoring the balance of mucin and water. Pharmacologic blockade of the epithelial $\mathrm{Na}^{+}$channel, which is rate-limiting for volume absorption from airway surfaces, constitutes a novel therapeutic target. Studies of mucus clearance both in sheep models and human subjects demonstrate that blockade of the epithelial $\mathrm{Na}^{+}$channel is associated with an acceleration of mucus clearance, suggesting that epithelial $\mathrm{Na}^{+}$channel blockade may indeed constitute a rational form of therapy for $\mathrm{CB}$.
\end{abstract}

Keywords: mucin epithelial sodium channel; airway surface liquid

Chronic bronchitis (CB) has been defined clinically as the presence of chronic cough and sputum production for 2 months in two consecutive years. The prevalence of CB in the United States is high, with in excess of 12-15 million Americans suffering from this syndrome. A general paradigm to describe the pathogenesis of genetic forms of $\mathrm{CB}$, for example, cystic fibrosis and primary ciliary dyskinesia, has been emerging. This paradigm focuses on the sequence of mucus obstruction, inflammation, and ultimately infection. The focus of this review is on the pathophysiology of mucus adhesion and airway obstruction and its possible contribution to the pathogenesis of acquired forms of $\mathrm{CB}$, for example, cigarette smoke-induced CB.

\section{MECHANISMS THAT LEAD TO FAILURE OF NORMAL MUCUS CLEARANCE}

Normal mucus clearance requires that the airway surfaces be covered by a liquid known as airway surface liquid (ASL), that contains the proper balance of mucin, salt, and water (1). ASL can be subdivided into a mucus layer and a periciliary liquid layer (Figure 1). The periciliary liquid layer (PCL) is a lowviscosity solution that allows cilia to beat efficiently and also acts as a lubricant layer for mucus clearance during cough. Overlying the PCL is the mucus layer, which functions to trap inhaled bacteria and also acts as a reservoir that can donate or accept liquid to or from the PCL as necessary. When these layers contain the proper ratios of mucin to salt and water, the cilia are able to move the entire ASL cephalad from the lung to the mouth

(Received in original form June 19, 2003; accepted in final form August 29, 2003)

Correspondence and requests for reprints should be addressed to Richard C. Boucher, M.D., University of North Carolina at Chapel Hill, Cystic Fibrosis/Pulmonary Research \& Treatment Center, CB\# 7248, 7011 Thurston Bowles, Chapel Hill, NC 27599-7248. E-mail: rboucher@med.unc.edu

Proc Am Thorac Soc Vol 1. pp 66-70, 2004

DOI: $10.1513 /$ pats. 2306018

Internet address: www.atsjournals.org
(2), effectively clearing materials that are trapped in ASL from airway surfaces.

CB may well be initiated by mucus stasis and adhesion. The general paradigm that leads to this functional defect is an imbalance of mucin, salt, and water on airway surfaces. Thus, CB is characterized by an excess of mucin compared with salt and water (hereafter referred to as "volume") that deranges the organization of ASL, impedes ciliary and cough-dependent clearance, and hence results in mucus stasis and obstruction.

Evidence for the notion that an imbalance of mucin and volume (salt and water) on airway surfaces leads to mucus stasis and obstruction has been slow to emerge because of difficulties in studying the morphology and function of the thin (less than $50 \mu \mathrm{m})$ ASL layer found in vivo. However, with the advent of the air-liquid interface, a well-differentiated culture system that exhibits not only the typical morphology of airway epithelia in vivo but also the ion and water transport, mucin secretion, and surface mucus transport properties of native epithelia, detailed studies have become feasible (2). In brief, this model system has allowed quantitative studies to be performed that allow the relationships between the mucin-to-volume ratios (indexed as percent solids content of the ASL) and surface mucus transport to be examined. Thus, studies in which volume is added to or subtracted from the ASL by varying the osmolality of the serosal bathing compartment have shown that mucus with a percent solids content that mimicks conditions in vivo, $2.5 \%$, was associated with a surface mucus transport rate of about $40 \mu \mathrm{m} / \mathrm{second}$, a value close to that observed in large airways in vivo (3). Increasing the percent solids content, that is, increasing the mucin concentration, from 2.5 to $6 \%$ virtually abolished surface mucus transport. Morphologic correlates of this reduction in mucus transport were the disappearance of the PCL, flattening of cilia, and adhesion of mucus to the glycocalyx lining the apical cell surface. In contrast, and somewhat surprisingly, a decrease in percent solids from the normal 2.5 to $1.5 \%$ was associated with a marked acceleration of mucus transport to about $80 \mu \mathrm{m} / \mathrm{second}$. The morphologic correlate of the addition of water to the ASL compartment was maintenance of the normal PCL height, that is, $7 \mu \mathrm{m}$, with a selective expansion of the mucus layer. This finding suggested that the mucus layer acts as a reservoir for liquid added to airway surfaces. It is not yet clear why mucus transport sped up with increased water content, with possibilities including an improvement of mucus layer viscoelastic properties and/or reduction of the viscosity of the PCL.

The imbalance between mucin and water, measured as an increase in percent solids, could lead to reduced mucus clearance in CB by at least two mechanisms that involve perturbations of the PCL. First, the efficacy of ciliary beat could be reduced by increased PCL viscosity. Second, the larger defect in clearance may reflect the adherence of a thickened mucus layer to airway surfaces. This adhesion event, reflecting the tethering of mucus to the cell surface, likely leads to the common clinical correlate of $\mathrm{CB}$; that is, the failure to clear retained mucus through coughdependent mechanisms. Thus, from a symptomatic point of view, it will be important to detach these adhesive mucus plaques/ plugs from airway surfaces so that they can be cleared from the lung. 


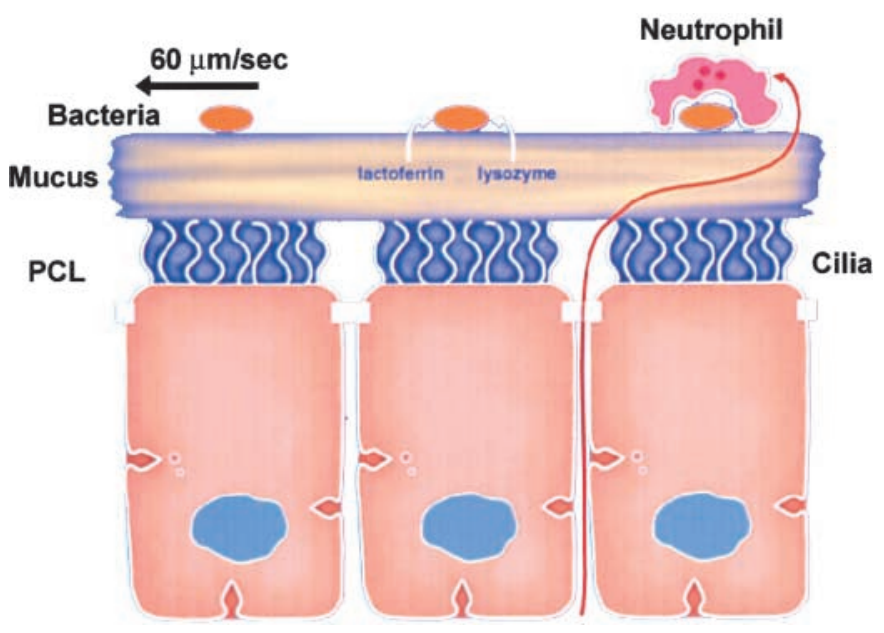

Figure 1. Normal mucus clearance. A mucus layer and periciliary liquid (PCL) layer are depicted. The PCL provides a low-viscosity solution for efficient ciliary beating and acts as a lubricant to prevent adhesion of the mucus layer to the cell surface.

\section{WHY IS MUCUS STASIS BAD?}

Mucus adherent to airway surfaces appears to be associated with most of the signs and symptoms of the syndrome of CB. First, as mucus accumulates on airway surfaces, local pressure and oscillations during airflow constitute irritants to airway surfaces, provoking cough. As noted above, this cough tends to be ineffective, that is, nonproductive, due to the adherence of the retained mucus mass/plaque to airway surfaces.

Second, it is probable that mucus stasis obstructs airflow. This concept has not been explored functionally as well as it might, because of the absence of agents that clear the lung rapidly of mucus.

Finally, retained mucus constitutes a nidus for infection. Indeed, it becomes difficult to develop the syndrome of infectious $\mathrm{CB}$ experimentally in vivo without mucus obstruction. At least two features of mucus stasis appear to contribute to the infectious phenotype. First, mucins are typically more than $80 \%$ carbohydrate, and many respiratory pathogens have developed the capacity to secrete extracellular "mucinases" to harvest the carbohydrates contained in mucin for growth. Second, as bacteria become enmeshed in thickened (greater than $6.5 \%$ solids) mucus, they become shielded from secondary airway defenses, for example, neutrophils. Thus, studies have shown that neutrophils can easily penetrate into 1.5 and $2.5 \%$ mucus gels, but not into $6.5 \%$ mucus gels (4). Further, neutrophils can penetrate 1.5 and $2.5 \%$ mucus gels to capture live bacteria to eradicate local infection. Conversely, when the mucus is thickened ( $6.5 \%$ or greater), the apparent pore size in the mucin mesh is so small that neutrophils cannot penetrate the mucus gel to capture or kill bacteria. Thus, a vicious cycle is set up whereby frustrated neutrophils release elastase and other extracellular killing factors that promote intraairway inflammation, further mucin secretion, and, ultimately, destruction of airway walls.

\section{ETIOLOGIES OF BRONCHITIC SYNDROME}

There are at least two genetic forms of $\mathrm{CB}$, primary ciliary dyskinesia and cystic fibrosis (CF). In addition, the most common form of $\mathrm{CB}$ in North America is the acquired form, which typically reflects cigarette smoking and, to a lesser degree, air pollution.
ASL
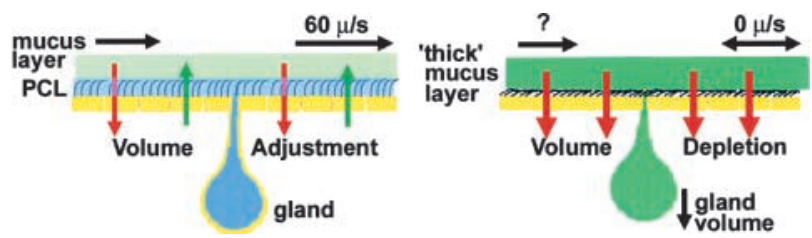

Figure 2. Airway surface liquid (ASL) depletion initiates cystic fibrosis (CF) lung disease. In normal airways (left), airway epithelia blend active $\mathrm{Na}^{+}$absorption (red arrows) and active $\mathrm{Cl}^{-}$secretion (green arrows) to fine-tune the PCL volume and promote mucus transport. In CF airway epithelia (right), unopposed and unregulated $\mathrm{Na}^{+}$absorption depletes $\mathrm{PCL}$, concentrates the mucus layer, and promotes adhesion of the mucus layer to the cell surface. These immobilized mucus plaques become the nidus for infection.

It is perhaps CF that is the most intensively studied form of CB. Although there has been debate in the literature, it appears that cystic fibrosis is a form of $\mathrm{CB}$ in which the imbalance between mucin and volume reflects a primary volume defect (see Figure 2). Thus, there are in CF combined defects in regulation of $\mathrm{Na}^{+}$transport rates and the initiation of $\mathrm{Cl}^{-}$secretion that lead to a failure to regulate ASL/PCL volume adequately on airway surfaces. Thus, early in the course of the disease, the imbalance between mucin and volume reflects a normal rate of mucin secretion into a diminished volume of ASL. Later in the course of the disease, with persistent inflammation and infection, mucin secretion is increased, which further exacerbates the imbalance between mucin and volume. It is likely that the severity of this mucin-volume imbalance is a prime determinant of the severity of cystic fibrosis airway disease.

In primary ciliary dyskinesia, the obvious problem in mucociliary clearance is the failure of cilia to beat properly. However, even in this disease, it is likely that there is an imbalance between mucin and volume. This imbalance occurs because there is normal mucin secretion into the lumen, but no mechanism for clearance of mucins via surface transport or transepithelial absorption. In contrast, the excess ASL volume that is retained on airway surfaces as a result of failure of ciliary activity can be absorbed as airway epithelia regulate electrolyte ion transport. Thus, even primary ciliary dyskinesia can be viewed in the context of mucus-volume imbalance.

Much less effort has been devoted to elucidating the pathogenesis of the mucus transport defects in cigarette smokinginduced $\mathrm{CB}$. There have been reports that cigarette smoke extracts can reduce the capacity of canine airway epithelia to secrete $\mathrm{Cl}^{-}$ ions, suggesting that there may be defects in volume on airway surfaces (5). However, studies of freshly excised human bronchi from cigarette smokers suggest that there are relatively few tonic effects on electrolyte transport properties (Table 1 [6]). Thus, whereas it is possible that there may be subtle defects in ASL volume, these data would argue for increased mucin relative to volume as the initiating event (Figure 3 ). This notion is consistent with the morphologic correlates of $\mathrm{CB}$, which demonstrates pronounced goblet cell hyperplasia. In vitro studies have suggested that a five- to sixfold increase in mucin secretion could be sufficient, in the face of normal electrolyte/volume transport, to increase percent solids to a level at which mucus transport may cease. This hypothesis rests on the notion that mucins are secreted into ASL "dry," that is, mucin-secreting cells selectively release mucins without associated salt and water. Although no direct studies have tested this notion, the concept is consistent with the segregation of ion transport elements, for example, the cystic fibrosis transmembrane conductance regulator, to ciliated cells, and the imaging studies that support the concept that mu- 
TABLE 1. BASAL BIOELECTRIC PROPERTIES AND ION FLOWS ACROSS BRONCHI FROM CIGARETTE SMOKERS, EX-SMOKERS, AND NONSMOKERS

\begin{tabular}{|c|c|c|c|}
\hline & $\begin{array}{c}\text { Nonsmokers } \\
\quad(n=6)\end{array}$ & $\begin{array}{c}\text { Ex-smokers } \\
\quad(n=9)\end{array}$ & $\begin{array}{l}\text { Smokers } \\
(n=25)\end{array}$ \\
\hline \multicolumn{4}{|c|}{ Bioelectric property } \\
\hline$I_{s c,} \mu A \cdot c^{-2}$ & $49.6 \pm 57$ & $53.1 \pm 4.3$ & $48.7 \pm 1.5$ \\
\hline $\mathrm{G}, \mathrm{mS} \cdot \mathrm{cm}^{-2}$ & $8.6 \pm 0.8$ & $9.9 \pm 0.6$ & $9.7 \pm 0.3$ \\
\hline \multicolumn{4}{|c|}{ Ion flow parameter } \\
\hline $\int_{\text {net }}^{\mathrm{Na}^{+}}$ & $1.63 \pm 0.46^{*}$ & $1.35 \pm 0.41^{*}$ & $1.53 \pm 0.21$ \\
\hline $\int_{n e t}^{C^{1-\dagger}-\dagger}$ & $-0.40 \pm 0.36$ & $-0.12 \pm 0.35$ & $0.20 \pm 0.26$ \\
\hline
\end{tabular}

Definition of abbreviations: $\mathrm{G}=$ conductance; $\mathrm{I}_{\mathrm{sc}}=$ short-circuit current; $\mathrm{J}_{\mathrm{net}}^{\mathrm{Cl}}=$ net flux of chloride; $\mathrm{Nat}_{\mathrm{nat}}^{\mathrm{N}}=$ net flux of sodium.

Reprinted by permission from Reference 6 .

Values represent means $\pm \mathrm{SE}$.

* Different from $0(p<0.05)$

${ }^{\dagger}$ Negative values for $J_{\text {net }}$ indicate secretion.

cins "swell," that is, hydrate themselves, as they enter the extracellular environment $(7,8)$.

From a clinical point of view, an increase in the mucin-tovolume ratio in ASL sufficient to produce mucus stasis may occur more focally in cigarette smoking-induced $\mathrm{CB}$ than in $\mathrm{CF}$. For example, it is well known that in cigarette smoking-induced CB there are focal areas of goblet cell hyperplasia, typically at airway bifurcations. Thus, one could speculate that the somewhat milder airway surface/infectious phenotype in cigarette smokinginduced $\mathrm{CB}$ would reflect the more focal nature of mucus adhesion and possibly the fact that the percent solids level does not rise to that seen in CF. However, it is important to stress that the genesis of mucus stasis does reflect the same basic pathogenesis, that is, an imbalance between mucin and volume that leads to adherence of mucus to airway surfaces.

It is important to note that the inflammation that is a hallmark of cigarette smoke-induced $\mathrm{CB}$ and infection likely would serve to exacerbate the problem of mucus adhesion and plugging. For example, serum proteins that leak into the lumen in response to inflammation may interact with mucins and increase their adhesive properties. Similarly, the DNA associated with neutrophil influx in response to inflammation and infection would also have an adverse impact on mucus adhesive properties.

\section{HOW CAN WE TREAT THE MUCUS STASIS COMPONENT OF CB?}

Ultimately, the preferred form of therapy is to treat the initiating event that leads to the imbalance between mucin and volume on chronic bronchitic airway surfaces. With respect to the genetic diseases primary ciliary dyskinesia and $\mathrm{CF}$, the rational way to restore the defective functions is through genetic correction approaches. However, the large numbers of studies designed to correct the CF defect in airway epithelial cells have by and large illustrated the difficulty in achieving efficient gene transfer in the airway lumenal environment (9-11). Approaches that will successfully evade the multiple barriers to gene transfer on airway surfaces, coupled with novel targeted vectors, should in future be able to cure these genetic forms of CB. In contrast, much of the syndrome of cigarette smoking-induced CB can be ameliorated by cessation of smoking. However, this is a difficult goal to achieve, and indeed, after a certain point, the airway damage inflicted by cigarette smoke becomes irreversible and the $\mathrm{CB}$ phenotype fixed. Even in these cases, however, there may be much to do pharmacologically to redress the imbalance of mucin and volume to restore the airway surfaces to health.

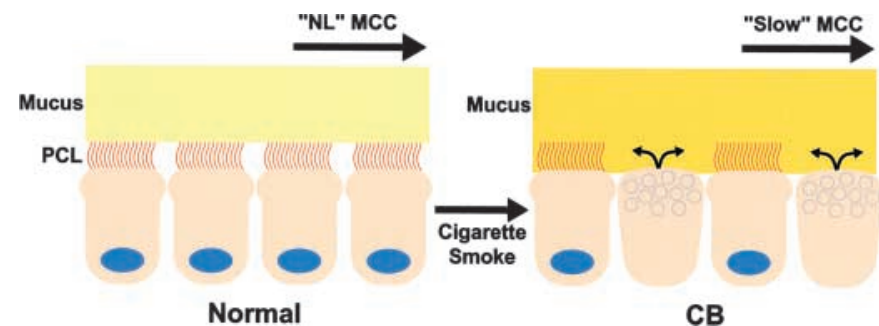

Figure 3. Mucus hypersecretion produces an imbalance in mucin-toairway surface liquid volume that slows mucociliary clearance (MCC) and produces mucus adhesion. As compared with normal ("NL") airway epithelia (left), cigarette smoke-induced chronic bronchitic (CB) airway epithelia (right) develop goblet cell hyperplasia and raised mucin secretion. The excess mucins "soak up" all available airway surface liquid, thus depleting PCL and promoting mucus adhesion to cell surfaces. Percent solids of mucus is accordingly raised in CB.

Two fundamentally different approaches can be envisioned to achieve this goal.

\section{Reduction of Mucin Secretion}

Certainly, in cigarette smoking-induced $\mathrm{CB}$, and in the later stages of cystic fibrosis, it may make sense to reduce the rate of mucin secretion into airways to "thin" mucus, that is, to restore the balance between mucin and volume. There are two ways this goal can be achieved. First, one can block the secretion of mucins from preexisting goblet cells. This goal theoretically can be achieved by blocking receptors that trigger mucin release. Currently, the most active cell surface receptor on goblet cells that triggers mucin secretion is the $\mathrm{P} 2 \mathrm{Y}_{2}$ receptor, and hence $\mathrm{P}_{2} \mathrm{Y}_{2}$ antagonists may be a rational choice for this purpose (7). Alternatively, one may try to block the secretory process more distally, and the recognition that the MARCKS (myristoylated alanine-rich $\mathrm{C}$ kinase substrate) protein is critical for vesicle fusion and release suggests that indeed this protein may be a rational target for prevention of mucin secretion (12). A theoretical problem with both approaches is that as mucin synthesis persists, goblet cells may become engorged with mucin. If there are transient periods during which block is not achieved or mechanisms evolve to circumvent the block, a dangerously high rate of mucin release might occur, perhaps mimicking the excessive release that occurs in status asthmaticus.

The alternative approach is to block the formation of new goblet cells, that is, block goblet cell hyperplasia. In the broadest approach, reduction of inflammation and infection should decrease goblet cell hyperplasia. More precise targets to achieve this goal will emerge from a more complete understanding of the mechanisms that lead to goblet cell metaplasia and hyperplasia. Currently, there are several approaches to achieving this goal. For example, there are relatively nonselective agents that may block goblet cell hyperplasia via interactions with the putative human calcium-activated $\mathrm{Cl}^{-}$channel hCLCA1 (13). An alternative, intriguing approach is to block the epidermal growth factor receptor pathway in airway epithelia. This approach rests on the hypothesis that the epidermal growth factor receptor can be activated by endogenous epidermal growth factor receptor-like ligands, perhaps contained in cell surface mucins, that produce goblet cell hyperplasia.

A question that persists with regard to both mechanisms, particularly with regard to cigarette smoking-induced $\mathrm{CB}$, is the long-term safety consequences of decreasing mucin secretion in the presence of inhalation of large quantities of toxicants. For example, it has been suggested that mucins provide an effective 

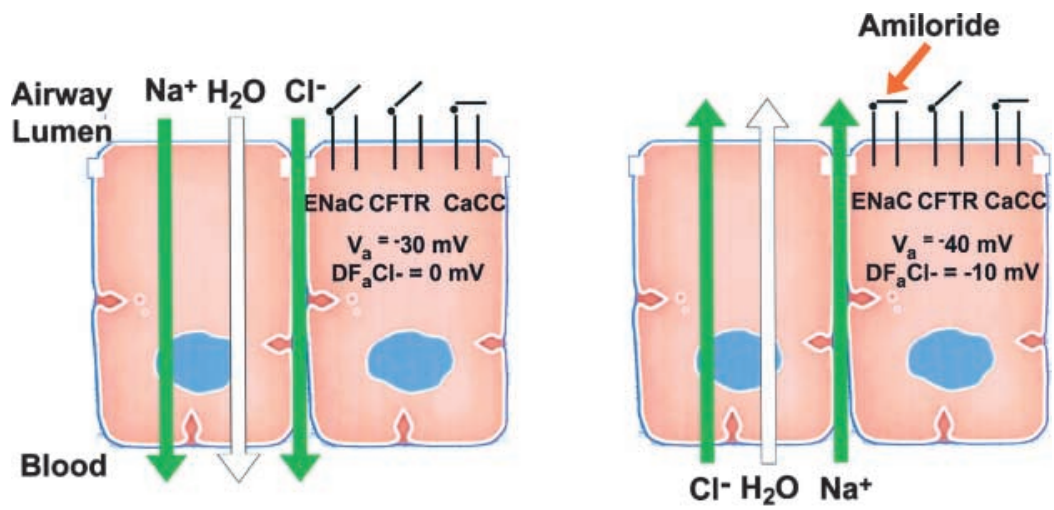

Figure 4. Model depicting the action of amiloride to induce volume secretion. Under basal conditions (left), airway epithelia absorb $\mathrm{Na}^{+}$transcellularly via the epithelial $\mathrm{Na}^{+}$channel (ENaC), whereas $\mathrm{Cl}^{-}$is absorbed passively via the paracellular path. After amiloride treatment (right), $\mathrm{Na}^{+}$ absorption is blocked and blockade of this conductance hyperpolarizes the apical membrane potential $\left(\mathrm{V}_{\mathrm{a}}\right)$ and generates an electrochemical driving force $\left(\mathrm{DF}_{\mathrm{a}}^{\mathrm{Cl}-}\right)$ to initiate transcellular $\mathrm{Cl}^{-}$secretion. $\mathrm{Na}^{+}$now follows $\mathrm{Cl}^{-}$via the paracellular path. Water follows salt transport in response to the osmotic gradient (white arrow). CFTR = cystic fibrosis transmembrane conductance regulator; $\mathrm{CaCC}=$ calciumactivated chloride channel. barrier to inhaled toxicants by binding them to prevent their penetration into airway epithelia. The demonstration that mice lacking MUC2 in the colon exhibited a spontaneous midlife colon carcinoma phenotype suggests that there could be longterm adverse events as a result of blocking adequate mucin secretion and prevention of toxin penetration into airway epithelia (14).

\section{Addition of Volume to Thin Mucus and Promotion of Clearance}

The concept that adding volume (salt and water) to mucus increases mucus transport has been explored in vitro (see above). However, there are no practical therapeutic agents designed to test the hypothesis that a strategy to restore mucus clearance by volume addition could be effective therapeutically. There are several reasons for the failure heretofore to test this concept.

First, many of the agents that have been touted to restore mucus clearance in clinical disease phenotypes, for example, cigarette smoking-induced $\mathrm{CB}$ and $\mathrm{CF}$, do not effectively add volume to airway secretions. As shown in Figure 4, the ability to add volume to airway secretions rests on the capacity to block the epithelial $\mathrm{Na}^{+}$channel. Typically, blockage of the epithelial $\mathrm{Na}^{+}$channel not only slows liquid absorption but also reverses the driving force for net ion flow to initiate $\mathrm{Cl}^{-}$secretion. Currently, only two classes of agents, $\mathrm{P}_{2} \mathrm{Y}_{2}$ agonists $(15,16)$ and amiloride-like drugs (17), have the capacity to block the epithelial $\mathrm{Na}^{+}$channel and reverse the gradients for ion transport. As shown in Figure 5, $\beta$ agonists do not inhibit epithelial $\mathrm{Na}^{+}$channel activity, but rather, selectively activate the cystic fibrosis transmembrane conductance regulator, which does not result in $\mathrm{Cl}^{-}$secretion or liquid addition. Thus, $\beta$ agonists are predicted to be progressively less effective in restoring mucociliary clearance as $\mathrm{CB}$ becomes more severe. It is, therefore, likely that the positive effects on mucociliary clearance in early cigarette smoking-induced $\mathrm{CB}$ disease are mediated by increases in ciliary beat frequency, but as the disease becomes more severe and mucociliary clearance is slowed by increased mucin-volume imbalances, $\beta$ agonists are ineffective because of their failure to add volume to airway surfaces.

The $\mathrm{P}_{2} \mathrm{Y}_{2}$ agonists have just begun to appear in clinical trials (18). In short-term studies, they do appear to be able to restore abnormal mucus clearance rates into the normal range (19). A theoretical question with regard to these agents is whether the combined actions on volume secretion and mucin secretion may offset each other.

The amiloride class of compounds has been extensively tested in the CF form of CB (17), but not in cigarette smoking-induced CB. Amiloride was found to be effective in acute studies designed to test for acute restoration of mucus clearance in $\mathrm{CF}$. However, the duration of the therapeutic effects on $\mathrm{Na}^{+}$channels to restore mucus transport were short, about 30 minutes, and it is probable that this pharmacodynamic property led to the failure of amiloride in chronic trials in adult patients with cystic fibrosis. Subsequent in vitro studies have revealed that the short duration of action of amiloride reflected the low potency of the compound ( $\mathrm{IC}_{50}$ about $1 \mu \mathrm{M}$ on human airway surfaces), the rapid absorption of amiloride from airway surfaces, and the rapid reversibility of the channel blockade.

A fundamental question is whether therapies designed to block $\mathrm{Na}^{+}$channels in the lung are safe. This issue was raised by studies of mouse models of $\mathrm{Na}^{+}$channel deletion in which the phenotype was neonatal lethality due to alveolar drowning (20). However, subsequent to those studies, clinical investigations of human patients with congenital loss-of-function mutations in epithelial $\mathrm{Na}^{+}$channel, "pseudohypoaldosteronism," revealed striking but different results (21). First, these patients
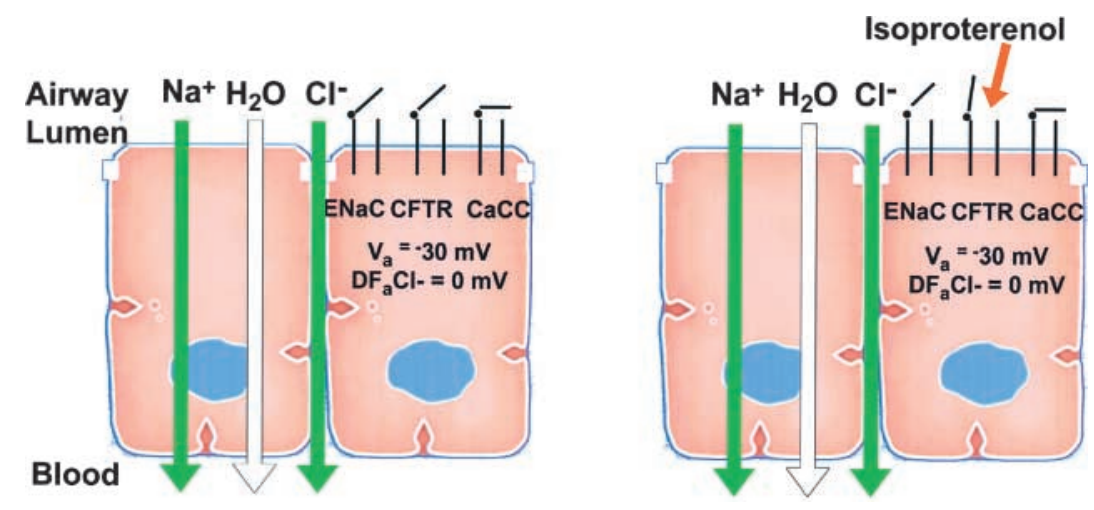

Figure 5. $\beta$ Agonists do not initiate volume secretion. Selective activation of the cystic fibrosis transmembrane conductance regulator (CFTR) by isoproterenol (right) does not inhibit the EnaC and consequently does not hyperpolarize $\mathrm{V}_{a}$, nor induce a $\mathrm{DF}_{\mathrm{a}}^{\mathrm{Cl}}$ for $\mathrm{Cl}^{-}$secretion. Therefore, no gradients are generated for volume secretion, and absorption persists. $\mathrm{CaCC}=$ calcium-activated chloride channel. 
exhibited a phenotype consistent with loss of transepithelial volume $\mathrm{Na}^{+}$-dependent absorption, that is, an increase in isotonic liquid on upper and lower airway surfaces. Second, these patients appeared to compensate for the absence of transepithelial volume absorption by accelerating the surface clearance of liquid from airways. Indeed, these patients had basal mucus transport rates that were the fastest observed, exceeding those of normal subjects after inhalation of $\beta$ agonists, purinoceptor agonists, or indeed, amiloride. Third, in adults, there was no disease phenotype associated with the absence of $\mathrm{Na}^{+}$channel blockade in the lung. For example, there was no evidence of airway infection or upper airway sinusitis. Notably, there was no evidence of alveolar flooding. Consequently, it appears that long-term $\mathrm{Na}^{+}$ channel blockade may be safe and effective in promoting clearance.

These considerations have led to programs to synthesize more potent and specific $\mathrm{Na}^{+}$channel blockers for inhalational use for therapy of CB. Compounds have now been synthesized that are 50- to 100-fold more potent than amiloride and 5- to 10 -fold less absorbable. Preclinical studies involving a sheep mucociliary clearance assay have led to observations that these pharmacologic properties lead to predictable pharmacodynamic increments in mucociliary clearance efficacy in sheep. For example, compounds that exhibit potencies 50- to 100 -fold greater than amiloride, and absorption rates equal to 1-10 times less than amiloride, accelerate mucus transport about 2-fold more than amiloride or $\beta$ agonists. Indeed, the efficacy of the newer compounds appears to approach that observed in humans with pseudohypoaldosteronism, suggesting that complete blockage can routinely accelerate mucus transport in normal subjects fourfold. Thus, these studies augur well that these agents will be, if anything, more effective in $\mathrm{CB}$, where the fold restoration of mucus clearance will be enhanced because of the basal reduction of clearance rates observed in these patients (22).

\section{Conclusions}

In conclusion, it appears that a concept has emerged to describe the pathogenesis of the mucosal surface phenotype associated with CB. An imbalance between mucin and volume depletes the periciliary liquid layer from airway surfaces and creates an adherent, tacky mucus layer. When the mucus layer adheres to the cell surface, both cilia-dependent and cough-dependent clearance mechanisms are deranged. Adherent mucus itself may be irritating and ultimately will become the nidus for bacterial infection that promotes inflammation in airway walls. Pharmacologic therapies to address this imbalance can be focused on reducing the rate of mucin secretion into the airway lumen, thereby "thinning" airway secretions. Alternatively, the addition of volume (salt and water) to airway secretions may be a safer way to clear the airways of mucins and the bound toxins/bacteria that, directly or through interactions with inflammatory cells, can damage the airway wall and lead to long-term adverse health events.

\section{References}

1. Knowles MR, Boucher RC. Mucus clearance as a primary innate defense mechanism for mammalian airways [Perspective]. J Clin Invest 2002; 109:571-577.
2. Matsui H, Randell SH, Peretti SW, Davis CW, Boucher RC. Coordinated clearance of periciliary liquid and mucus from airway surfaces. J Clin Invest 1998;102:1125-1131.

3. Tarran R, Grubb BR, Gatzy JT, Davis CW, Boucher RC. The relative roles of passive surface forces and active ion transport in the modulation of airway surface liquid volume and composition. J Gen Physiol 2001;118:223-236.

4. Matsui H, Verghese MW, Boucher RC. Low motility in thick mucus prevents neutrophils from catching bacteria [abstract]. Pediatr Pulmonol 2002;265.

5. Welsh MJ. Cigarette smoke inhibition of ion transport in canine tracheal epithelium. J Clin Invest 1983;71:1614-1623.

6. Knowles M, Murray G, Shallal J, Askin F, Ranga V, Gatzy J, Boucher R. Bioelectric properties and ion flow across excised human bronchi. J Appl Physiol 1984;56:868-877.

7. Davis CW, Dowell ML, Lethem MI, Van Scott M. Goblet cell degranulation in isolated canine tracheal epithelium: response to exogenous ATP, ADP, and adenosine. Am J Physiol 1992;262:C1313-C1323.

8. Verdugo P. Hydration kinetics of exocytosed mucins in cultured secretory cells of the rabbit trachea: a new model. In: Nugent J, O'Connor M, editors. Mucus and mucosa. London: Pitman; 1984. p. 212-225.

9. Johnson LG. A perspective on gene therapy for cystic fibrosis. Exp Opin Invest Drugs 1996;5:29-42.

10. Johnson LG. Gene therapy for cystic fibrosis. Chest 1995;107(2 Suppl): $77 \mathrm{~S}-83 \mathrm{~S}$.

11. Ferrari S, Geddes DM, Alton EW. Barriers to and new approaches for gene therapy and gene delivery in cystic fibrosis. Adv Drug Deliv Rev 2002;54:1373-1393.

12. Li Y, Martin LD, Spizz G, Adler KB. MARCKS protein is a key molecule regulating mucin secretion by human airway epithelial cells in vitro. J Biol Chem 2001;276:40982-40990.

13. Toda M, Tulic MK, Levitt RC, Hamid Q. A calcium-activated chloride channel (HCLCA1) is strongly related to IL-9 expression and mucus production in bronchial epithelium of patients with asthma. $J$ Allergy Clin Immunol 2002;109:246-250.

14. Velcich A, Yang W, Heyer J, Fragale A, Nicholas C, Viani S, Kucherlapati R, Lipkin M, Yang K, Augenlicht L. Colorectal cancer in mice genetically deficient in the mucin Muc2. Science 2002;295:1726-1729.

15. Devor DC, Bridges RJ, Pilewski JM. Pharmacological modulation of ion transport across wild-type and $\Delta$ F508 CFTR-expressing human bronchial epithelia. Am J Physiol 2000;279:C461-C479.

16. Mall M, Wissner A, Gonska T, Calenborn D, Kuehr J, Brandis M, Kunzelmann K. Inhibition of amiloride-sensitive epithelial $\mathrm{Na}^{+}$absorption by extracellular nucleotides in human normal and cystic fibrosis airways. Am J Respir Cell Mol Biol 2000;23:755-761.

17. Hirsh AJ. Altering airway surface liquid volume: inhalation therapy with amiloride and hyperosmotic agents. Adv Drug Deliv Rev 2002;54:14451462.

18. Kellerman D, Evans R, Mathews D, Shaffer C. Altering airway surface liquid volume: inhalation therapy with amiloride and hyperosmotic agents. Adv Drug Deliv Rev 2002;54:1463-1474.

19. Olivier KN, Bennett WD, Hohneker KW, Zeman KL, Edwards LJ, Boucher RC, Knowles MR. Acute safety and effects on mucociliary clearance of aerosolized uridine 5 '-triphosphate $+/-$ amiloride in normal human adults. Am J Respir Crit Care Med 1996;154:217-223.

20. Hummler E, Barker P, Gatzy J, Beermann F, Verdumo C, Schmidt A, Boucher R, Rossier BC. Early death due to defective neonatal lung liquid clearance in $\alpha$-ENaC-deficient mice. Nat Genet 1996;12:325-328.

21. Kerem E, Bistritzer T, Hanukoglu A, Hofmann T, Zhou Z, Bennett W, MacLaughlin E, Barker P, Nash M, Quittell L, et al. Pulmonary epithelial sodium channel dysfunction and excess airway liquid in pseudohypoaldosteronism. N Engl J Med 1999;341:156-162.

22. Wanner A, Salathe M, O'Riordan TG. Mucociliary clearance in the airways. Am J Respir Crit Care Med 1996;154:1868-1902. 